\title{
Sexual Intercourse and the Age Difference Between Adolescent Females and Their Romantic Partners
}

\author{
ByChristine E. \\ Kaestle, Donald E. \\ Morisky and \\ Dorothy J. Wiley
}

Christine E. Kaestle is a doctoral student, Department of Maternal and Child Health,

University of North Carolina, Chapel Hill, NC. Donald E.

Morisky is professor, Department of Community Health Sciences, and Dorothy J. Wiley is assistant professor, Division of Primary Care, School of Nursing, University of California at Los Angeles.

CONTEXT: The age difference between a female and her partner may influence relationship dynamics in ways that put the female at increased risk of unintended pregnancies and sexually transmitted diseases. Very little is known, however, about how romantic involvement progresses to intercourse, particularly among adolescent females with older male partners.

METHODS: Data from 1,975 female participants in the National Longitudinal Study of Adolescent Health were analyzed using logistic regression to determine whether the age difference between an adolescent female and her romantic partner is a risk factor for sexual intercourse.

RESULTS: Adolescent females involved with an older partner have higher odds of having intercourse with that partner than females with partners their own age, after adjustment for demographic covariates. The magnitude of this association is most dramatic among the youngest females - for example, the odds of intercourse among 13-year-old females with a partner six years older are more than six times the odds among 13-year-old females with a same-age partner (odds ratio, 6.4), while 17-year-old females with partners six years older have about twice the odds of intercourse when compared with those who have a same-age partner (2.1).

CONCLUSIONS: Young adolescent females with substantially older partners are much more likely than their peers to have sex with their partner, which exposes them to the risks of pregnancy and sexually transmitted diseases.

Perspectives on Sexual and Reproductive Health, 2002, 34(6):304-309

Researchers and policymakers have focused concern and attention on adolescent females who have romantic relationships with older males. The age difference between a female and her partner may influence relationship dynamics in ways that put the female at greater risk of both unintended pregnancies and sexually transmitted diseases (STDs). However, our current understanding of the role of age gaps in romantic relationships is limited, and it is based mainly on studies of couples who are already sexually active. We address this limitation by investigating how the age difference between partners is related to whether a romantic relationship includes sexual intercourse.

\section{AGE GAP AND SEXUAL BEHAVIOR}

Having an older partner is associated with early sexual debut. In a survey of sixth graders, students who reported ever having had a boyfriend or girlfriend at least two years older had more than 30 times the odds of those who had never had a boyfriend or girlfriend of having had sex. The survey, however, did not specify whether sex had occurred with the older partner, and results did not differ between students with an older partner and those with a same-age partner. ${ }^{1}$ Age gaps between partners also influence contraceptive use: An analysis of data from the National Longitudinal Study of Adolescent Health (Add Health) showed that adolescents with a sexual partner who was older by more than two years had a reduced likelihood of using condoms. ${ }^{2}$
Females have a higher risk than males of contracting STDs during sexual intercourse with an infected partner, and the health risks associated with an unplanned pregnancy are borne by females. For these and other reasons, many studies of the effect of age difference on sexual behavior have focused on the experiences of adolescent and young adult women. ${ }^{3}$ For example, Darroch and colleagues examined data from the 1995 National Survey of Family Growth (NSFG) and found that the pregnancy rate for females whose sexual partners were older by six or more years was 3.7 times the rate for females whose partners were within two years of their age. ${ }^{4}$ Using data from the same survey, Glei found that females aged 15-17 whose partner was three or more years older were 33\% less likely than those with partners closer in age to use contraceptives; in contrast, among females 18 and older, having an older partner had little effect on contraceptive use. ${ }^{5}$

Two studies have investigated the association between age difference and aspects of females' sexual debut. Abma and colleagues used 1995 NSFG data to examine partner age difference in the context of measuring "wantedness" of first intercourse. Their findings suggested that wantedness is inversely related to the age difference between a young woman and her partner, and that it may be related to her limited control over the situation. ${ }^{6}$ Furthermore, Leitenberg and Saltzman found that among females who had had first sexual intercourse at ages 11-12, those who had 
a partner five or more years their senior had an elevated risk of attempted suicide, substance abuse and pregnancy. Among females who had had first intercourse between the ages of 13 and 15, having older partners was associated only with truancy. In contrast, among females whose age at first intercourse was 16-18, having older partners was not linked to these negative outcomes. Thus, adverse effects of partner age difference may lessen as females mature. ${ }^{7}$

Particular demographic characteristics are associated with sexual behavior among adolescents and may be associated differentially with age gap between a female and her romantic partner. ${ }^{8}$ To better understand the link between age gap and sexual intercourse, to adjust for confounding and to examine how relationships among adolescents may be influenced by demographic characteristics, we considered age, race and ethnicity, religion and family characteristics in analyses of a nationally representative sample of American adolescent females who had a romantic partner.

\section{METHODS}

Data

Add Health is a probability-based, nationally representative survey of U.S. adolescents who were enrolled in grades 7-12 in the 1994-1995 school year. ${ }^{9}$ Participants completed an in-home questionnaire that asked for demographic information, as well as information about health behaviors, community characteristics and health status. The first wave of Add Health was conducted by the University of North Carolina at Chapel Hill and is described in detail elsewhere. ${ }^{10}$

We used the publicly available version of results from the first wave of Add Health, which contained data on 6,504 adolescents and 2,801 variables. ${ }^{11}$ Of a total of 3,356 female respondents, 2,131 indicated that they had had a male romantic partner, defined as the first male that the respondent listed when asked, "In the last 18 months have you had a special romantic relationship with anyone?"* Older females were more likely to report a romantic relationship: For example, $80 \%$ of females who were 17 or older reported a romantic partner, compared with $65 \%$ of those aged $15-16$ and $45 \%$ of those aged 14 or younger.

Complete data for the variables that were relevant to our study were available for 1,975 females who reported having had a male romantic partner. Of the 156 females excluded because of incomplete records, $53 \%$ had missing data on religion, mother's education or partner's age; for the remainder, we could not estimate the age at which the romantic relationship began. Excluded females for whom we could estimate this age tended to be slightly younger than females in the study group (mean age, 14.3 vs. 14.7). However, the age gaps between excluded females and their partners were no different from those between study group respondents and their partners.

\section{Measures}

The outcome of interest, which we treated as a dichotomous variable, was whether a respondent had had intercourse with her romantic partner. Reports of intercourse were ver- ified by analyzing responses to the question "When you had sexual intercourse with [your partner], did he insert his penis into your vagina?"

The main independent variable of interest was the difference in age between a respondent and her male partner, which we treated as a continuous variable (coded in single years). We estimated the respondent's age at the start of the romantic relationship by calculating the interval between the month and year of her birth and the month and year during which the relationship began. We then calculated the difference between a respondent's age and the age of the partner (as reported by the respondent); a positive value indicated an older male partner, and a negative value a younger partner.

Other covariates that we examined were the respondent's age at the start of the romantic relationship (coded in single years), her race or ethnicity (coded as white, black, Hispanic or other), her religious affiliation (coded as Baptist, Catholic, none or other), her mother's educational level (whether the mother had graduated from college) and whether a "mother figure" lived in her home at the time of the interview. For these analyses, the definition of a mother or mother figure was not confined to the respondent's biological mother. Although of interest to us, it was not feasible to directly estimate socioeconomic status from questionnaire data; information about household income was often missing.

\section{Statistical Analyses}

We used STATA version 6 to adjust for the clustered sample design and to standardize demographic estimates of adolescents to U.S. Census Bureau estimates, as recommended by the Carolina Population Center. ${ }^{12}$ We used maximum likelihood multivariate logistic regression to model the relationship between partner age gap and the odds that a respondent had had intercourse with her partner, as well as to adjust odds ratios for the effects of demographic covariates. ${ }^{13}$ The cutoff level for significance was taken as $\mathrm{p} \leq .05$.

When a female begins a relationship with a new partner, the relationship may either progress to sexual intercourse or end before the couple has engaged in intercourse. Some respondents, however, reported an ongoing romantic relationship. Although intercourse might not have occurred by the interview date, it may have done so after the study, thereby censoring data for these relationships. In addition, because respondents may have been likely to list their current relationship first, reporting may have been biased toward ongoing relationships. To explore the effects of these potential biases, we repeated some of our analyses using data from the 993 respondents who reported only relationships that had already ended. In this way, the outcome of their relationship (intercourse or no intercourse) was firmly established.

*Respondents were asked to identify as many as three romantic partners
during the 18 months before the study, but fewer than $20 \%$ of respondents
identified more than one partner. Subsequent questionnaire items asked for characteristics of each partner in turn. 
TABLE 1. Percentage distribution of adolescent females with a romantic partner, by selected characteristics, National Longitudinal Study of Adolescent Health, 1995

\begin{tabular}{ll} 
Characteristic & $\%$ \\
& $(\mathrm{~N}=1,975)$ \\
\hline Age at start of relationship & \\
$\leq 13$ & 25.8 \\
14 & 18.8 \\
15 & 20.8 \\
16 & 19.9 \\
$\geq 17$ & 14.7 \\
Partner age & \\
Younger/same age* & \\
1 yr. older & 28.2 \\
$2-3$ yrs. older & 23.7 \\
$4-5$ yrs. older & 32.6 \\
$\geq 6$ yrs. older & 10.2 \\
Race/ethnicity & 5.3 \\
White & \\
Black & \\
Hispanic & 68.5 \\
Other & 15.0 \\
Religion & 10.6 \\
Baptist & 6.0 \\
Catholic & \\
Other & \\
None & 23.7 \\
Mother figure at home & 24.0 \\
Yes & 40.8 \\
No & 11.5 \\
Mother's education & \\
<college graduate & \\
College graduate & 93.8 \\
Total & 6.2 \\
\hline *Includes partners older by less than one year. Note: Percentages are weight- \\
ed; Nis unweighted. & \\
& \\
& \\
& \\
& \\
& \\
&
\end{tabular}

\section{RESULTS}

\section{Descriptive Analyses}

Most survey respondents were younger than 17 at the start of their relationship, had a partner who was older by $1-5$ years, were white and had a resident mother figure who was not a college graduate (Table 1). Respondents' ages at the start of the relationship ranged from seven to 19 years, and their mean and median ages were about 15 years (not shown).

The differences in age between a female and her romantic partner ranged from less than five to more than 28 years. Partners' ages ranged from eight to 44 years, and the mean and median ages were $16-17$ years. Nearly $10 \%$ of the partners were 20 or older, and 90\% were in their teens. Just 7\% of adolescent females had a partner who was younger, mostly by one year (not shown). Partnerships with same-age or younger males were less common among females aged 13 or younger than among older females (16\% vs. $28-37 \%-$ Table 2).

Nearly one-half of respondents (43\%) reported having had sexual intercourse with their romantic partner, indicating that having a romantic relationship involves a high risk of sexual activity. The proportion of females reporting that they had had intercourse with their partner increased with age-from $24 \%$ among females aged 13 or younger at the start of the relationship to $41 \%$ among those aged 14-15 and $59 \%$ among those aged 16 or older (Table 2). The proportion reporting intercourse within each age-group also increased with partner age gap; this effect was smallest among the oldest respondents. Females aged 13 or younger with a partner at least four years their senior were more likely to have had sex than were those with a partner of the same age or younger (41\% vs. 9\%). Females aged at least 16 showed a similar trend, but the relative difference in the proportions of those having had intercourse was substantially smaller (68\% vs. 55\%). It thus seems that while sexual intercourse is less common for females who are 13 or younger at the onset of their relationships, substantial age gaps between partners may play a larger role in the progression to intercourse in this age-group than in others.

\section{Multivariate Analyses}

We estimated the effect of age gap on the odds of intercourse among females, using multivariate logistic regression to adjust for their age, race, religion, having a mother figure at home and mother's education. After adjustment for other demographic covariates, this analysis indicated that black females were more likely than white females to have had sex with their romantic partner (odds ratio, 1.7; 95\% confidence interval, 1.3-2.2), and females who did not live with a mother figure were more likely than those who did to have had sex with their partner (2.4; 95\% confidence interval, 1.5-3.8). Respondents whose mother had graduated from college had lower odds of having had sex with a romantic partner than those whose mother had not graduated (0.7; 95\% confidence interval, 0.5-0.9).

The analyses also revealed significant associations between sexual intercourse and both respondent's age and the age difference between partners. However, we found a significant interaction between these two variables, which persisted even after adjustment for the effect of the other

TABLE 2. Percentage distribution of adolescent females with a romantic partner, and percentage who have had intercourse with that partner, by age gap between partners, according to female's age at start of relationship

\begin{tabular}{lcc} 
Age and age gap & All & Have had intercourse \\
\hline Females aged $\leq \mathbf{1 3}$ & $(\mathrm{N}=485)$ & $(\mathrm{N}=131)$ \\
Partner younger/same age* & 16.4 & 8.5 \\
Partner older by 1-3 yrs. & 63.6 & 23.3 \\
Partner older by $\geq 4$ yrs. & 19.9 & 40.8 \\
Total & 100.0 & 24.3 \\
& & \\
Females aged 14-15 & $(\mathrm{N}=794)$ & $(\mathrm{N}=327)$ \\
Partner younger/same age* & 27.7 & 23.1 \\
Partner older by 1-3 yrs. & 59.3 & 42.9 \\
Partner older by $\geq 4$ yrs. & 13.1 & 67.4 \\
Total & 100.0 & 40.6 \\
& & \\
Females aged $\geq 16$ & $(\mathrm{~N}=696)$ & $(\mathrm{N}=399)$ \\
Partner younger/same age* & 37.4 & 55.0 \\
Partner older by 1-3 yrs. & 47.6 & 59.0 \\
Partner older by $\geq 4$ yrs. & 15.0 & 67.9 \\
Total & 100.0 & 58.8
\end{tabular}

*Includes partners older by less than one year. Note: Percentages are weighted; Ns are unweighted. 


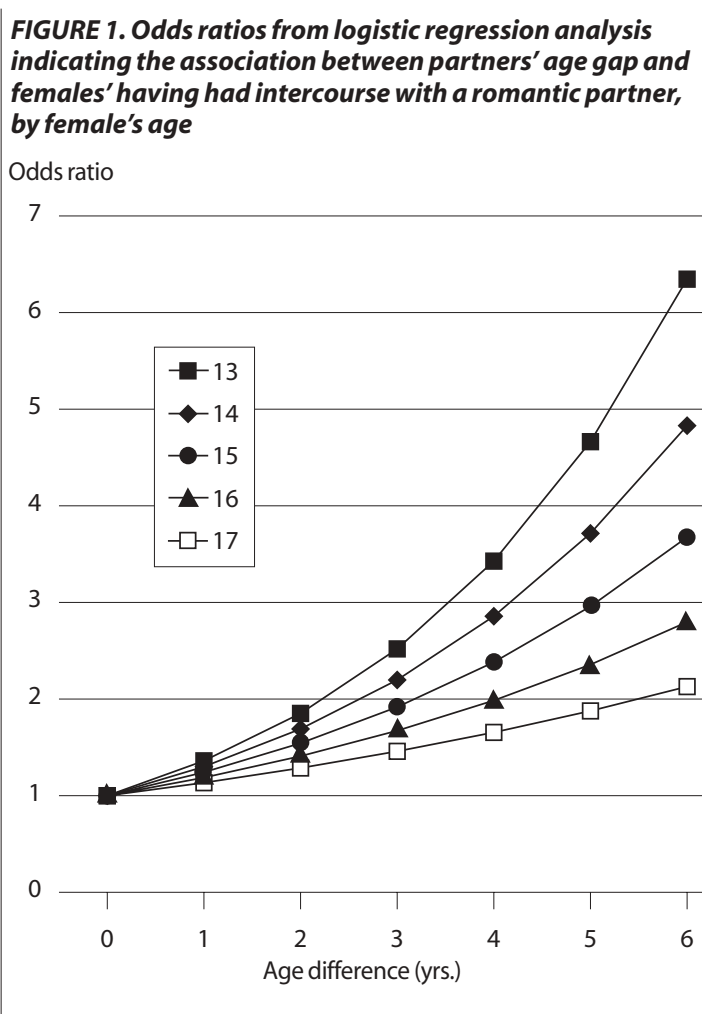

Notes: Odds ratios were adjusted for respondent's race or ethnicity, respondent's religion, presence of mother figure at home and mother's education. The reference for each age was the group of females with a same-age partner.

covariates. Because of this interaction, the effect of age gap on the odds of intercourse should be considered for each age of female separately.

To illustrate more clearly the effect of a female's age and age gap on the odds of intercourse, we calculated the odds of intercourse among females with older partners, relative to those among females with a same-age partner, by age gap and for each single year of age. As age difference increased, so did the odds of having engaged in sexual intercourse; this effect was strongest for the youngest females (Figure 1). For example, among 13-year-old females, the odds of intercourse with a partner who was six years older were more than six times the odds of intercourse with a sameage partner (odds ratio, 6.4; 95\% confidence interval, 3.4-11.8). In contrast, the odds among 17-year-old females with a partner six years their senior were about twice those among 17-year-old females with a same-age partner (2.1; 95\% confidence interval, 1.4-3.3).

When we limited our analyses to the 993 respondents who reported that their romantic relationship had ended, the association between age difference and the odds of having had intercourse was stronger than that found in the full model. For example, 13-year-old females with a partner six years older had odds of intercourse that were nearly 10 times those among 13-year-olds with same-age partners (odds ratio, 9.9; 95\% confidence interval, 5.0-19.9), and the odds among 17-year-olds with partners six years older were more than twice those of 17-year-olds with same-age partners (2.5; $95 \%$ confidence interval, 1.2-5.1). Thus, analysis of the full model, which includes both ended and ongoing relationships, reflects a more conservative estimate of the impact of partner age difference on the likelihood of sex with a romantic partner.

\section{DISCUSSION}

Our analyses suggest that the age difference between romantic partners is an important predictor of whether they will engage in sexual intercourse. Compared with females with same-age partners, those who had older partners had a higher risk of having had sexual intercourse. The magnitude of this association was most dramatic among the youngest adolescent females. It is important to keep in mind, however, that older females were more likely than younger females to have had intercourse. Therefore, both the age of an adolescent female and the age of her older partner have an important influence on whether a romantic relationship includes intercourse.

Age differences between partners may influence the progression of a romantic relationship in a variety of ways that would affect sexual activity. Older males may enter a romantic relationship with different expectations from younger males about relationship behaviors, including greater expectations of sexual intercourse. Furthermore, females who become involved with older partners may experience longer relationships because older males may expect or desire longer or more "serious" relationships than same-age males; one result may be a higher likelihood of progression to intercourse. For this reason, we did not treat the duration of the relationship as a confounder; instead, we treated it as a possible intermediate factor in the pathway.

Although we could not directly estimate and control for respondents' socioeconomic status, our analyses may have controlled for some characteristics that are broadly associated with socioeconomic status: The observed associations of race or ethnicity and of mother's educational level with the odds of intercourse are likely driven largely by socioeconomic factors

The results largely agree with the findings of others that indicate an association between age gap and sexual behaviors among adolescent couples. ${ }^{14}$ Our finding of an interaction between a female's age and the age difference between partners is consistent with an earlier study showing that the effect of the age gap between a female and her first sexual partner on certain problem behaviors (suicide attempts, substance abuse and pregnancy) was largest among younger females. ${ }^{15}$ We suggest that power and communication dynamics between adolescent females and older partners may be substantially different for females in early and later adolescence. The data, however, did not allow us to determine whether females with older romantic partners specifically sought these relationships out of a desire for sexual activity or whether the older partner differentially pressured these females into having intercourse.

Our findings should be interpreted with caution. Although they support the idea that a romantic relationship between a young adolescent female and an older male is 
risky, we cannot determine the causal nature of the association. The findings are based on relationships that were identified by the respondents as "romantic," which may have been interpreted differently by respondents of different ages and within a given age-group. In addition, the romantic partner on whom our analyses are based was the first partner listed by the respondent, but we cannot determine why the respondent listed him first. Finally, these findings should not be extrapolated to relationships in which the male is substantially younger than the female.

Our results have important ramifications for public health intervention, especially regarding the risk of early sexual debut among America's youngest adolescent females when they have romantic relationships with older males. Public health programs often focus on changing females' behavior rather than changing the behaviors of older partners or addressing the factors that contribute to each partner's involvement in a relationship. They also rarely concentrate on the influence that parents might have on a young daughter's romantic associations. Although communication between parents and their daughters is complex, we believe that it can have a positive influence on how females choose partners at an early age. Parents-and teachers, pediatricians, gynecologists, school psychologists, nurses and other practitioners who work with young females-should be encouraged to communicate with young females about the age of their boyfriends.

Health education programs for parents of adolescents might focus on improving parenting and communication skills, as well as on parents' knowledge, perceptions and beliefs about characteristics of appropriate romantic partners for their child, no matter the child's age. For example, parents of male adolescents should be encouraged to communicate with their sons about healthy relationships and partner choices, to help them develop into men who are comfortable with and, consequently, pursue age-appropriate partners.

Sexuality and health education programs in schools should be designed with the realization that they may be reaching only half of a couple that is at high risk for sexual activity. New outreach efforts, settings and program structures may be required to reach adult male partners. ${ }^{16}$ In addition, because adolescent females who participate in education programs may not have the same power or influence in their relationship as their older partners, they may need help in identifying their own interests and in asserting their decisions. Simply educating females about abstinence and safer sexual practices is likely to be insufficient if their romantic partners are significantly older. Educational materials that directly address the issue of dating older males may be helpful, especially for younger adolescent females.

Our findings also may have special significance to policymakers with reference to statutory rape laws. For example, the relationship between a 13-year-old female and an older male may be qualitatively different than that between a 13-year-old female and a male of the same age. Similarly, a relationship between a 17 -year-old female and an older male partner is likely quite different from that between a 13-year-old female and an adult male. Statutory rape laws may deter older males from exploiting very young adolescent females. However, many statutory rape laws are written so broadly that full enforcement would involve the prosecution of same-age and slightly older male partners, who make up the majority of young females' sexual partners. ${ }^{17}$ In addition, the enforcement of statutory rape laws could create problems in the delivery of health, family planning and social services, because young women might fear that disclosing partner information to providers will put their partners at risk of prosecution. ${ }^{18}$ It may be possible to revise such laws to focus on situations in which partner age disparity is great, especially among very young females. ${ }^{19}$ Training may be helpful to law enforcement personnel who must enforce these laws; our findings suggest that intervention among the youngest adolescent females may have the greatest impact on risk of sexual intercourse.

Future research activities need to examine the reasons that adolescent females become involved with older partners, and vice versa, as well as the factors that promote sameage relationships between adolescents, which are less likely to be sexual. There is still much to learn about the timing and the quality and quantity of particular behavior patterns occurring in romantic relationships across the age spectrum of young teenagers (e.g., dating, meeting parents, kissing, heavy petting, intercourse and pregnancy). Research focusing on how age differences affect the behavior of both adolescent and young adult males is also warranted, and a greater effort should be made to understand older male partners' perceptions, characteristics and behaviors as targets for possible intervention. Furthermore, the full potential of parental involvement and monitoring on promoting ageappropriate partner choices is not known. We also need to better understand the circumstances under which statutory rape laws are enforced and the long-term effects of enforcement on females and their partners. Finally, health researchers who study sexual behavior in relation to a series of individual characteristics rather than within the context of relationship characteristics may be missing important predictors that are amenable to intervention.

\section{REFERENCES}

1. VanOss Marín B et al., Older boyfriends and girlfriends increase risk of sexual initiation in young adolescents, Journal of Adolescent Health, 2000, 27(6):409-418

2. Ford K, Sohn W and Lepkowski J, Characteristics of adolescents sexual partners and their association with use of condoms and other methods, Family Planning Perspectives, 2001, 33(3):100-105 \& 132

3. Leitenberg $\mathrm{H}$ and Saltzman H, A statewide survey of age at first intercourse for adolescent females and age of their male partners: relation to other risk behaviors and statutory rape implications, Archives of Sexual Behavior, 2000, 29(3):203-215; Glei DA, Measuring contraceptive use patterns among teenage and adult women, Family Planning Perspectives, 1999, 31(2):73-80; Abma J, Driscoll A and Moore K, Young women's degree of control over first intercourse: an exploratory analysis, Family Planning Perspectives, 1998, 30(1):12-18; and Lindberg LD et al., Age differences between minors who give birth and their adult partners, Family Planning Perspectives, 1997, 29(2):61-66. 
4. Darroch JE, Landry DJ and Oslak S, Age differences between sexual partners in the United States, Family Planning Perspectives, 1999 , 31(4):160-167.

5. Glei DA, 1999, op. cit. (see reference 3).

6. Abma J, Driscoll A and Moore K, 1998, op. cit. (see reference 3).

7. Leitenberg H and Saltzman H, 2000, op. cit. (see reference 3).

8. Kann L et al., Youth risk behavior surveillance-United States, 1997. State and local YRBSS coordinators, Journal of School Health, 1998, 68(9):355-369; Lammers C et al., Influences on adolescents' decision to postpone onset of sexual intercourse: a survival analysis of virginity among youths aged 13 to 18 years, Journal of Adolescent Health, 2000, 26(1):42-48; and Darroch JE, Landry DJ and Oslak S, 1999, op. cit. (see reference 4 ).

9. Udry JR, The National Longitudinal Study of Adolescent Health (Add Health), Waves I \& II, 1994-1996 [Machine-Readable Data File and Documentation], Chapel Hill, NC, and Los Altos, CA: Carolina Population Center and Sociometrics, 1998.

10. Sieving RE et al., Development of adolescent self-report measures from the National Longitudinal Study of Adolescent Health, Journal of Adolescent Health, 2001, 28(1):73-81; and Halpern CT et al., Smart teens don't have sex (or kiss much either), Journal of Adolescent Health, 2000, 26(3):213-225.

11. Lang E, McKean E and Peterson J, National Longitudinal Study of Adolescent Health, Wave 1: User's Manual for the CD-ROM, Los Altos, CA: Sociometrics, 1997.

12. Chantala K and Tabor J, National Longitudinal Study of Adolescen Health: Strategies to Perform a Design-Based Analysis Using the Add Health Data, Chapel Hill, NC: Carolina Population Center, 1999; and STATA Version 6 Statistical Software, College Station, TX: STATA.
13. Hosmer DW and Lemeshow S, Applied Logistic Regression, New York: Wiley, 1989

14. Darroch JE, Landry DJ and Oslak S, 1999, op. cit. (see reference 4); Glei DA, 1999, op. cit. (see reference 3); and Ford K, Sohn W and Lepkowski J, 2001, op. cit. (see reference 2).

15. Leitenberg H and Saltzman H, 2000, op. cit. (see reference 3).

16. Landry DJ and Forrest JD, How old are U.S. fathers? Family Planning Perspectives, 1995, 27(4):159-161 \& 165; and Lindberg LD et al. 1997 , op. cit. (see reference 3)

17. Leitenberg H and Saltzman H, 2000, op. cit. (see reference 3).

18. Donovan P, Can statutory rape laws be effective in preventing adolescent pregnancy? Family Planning Perspectives, 1997, 29(1):30-34 \& 40.

19. Leitenberg H and Saltzman H, 2000, op. cit. (see reference 3).

\section{Acknowledgments}

The authors thank the American Family Data Archive, Sociometrics Corp., Los Altos, CA, for providing data on which this study was based. Add Health was conducted by J. Richard Udry of the Carolina Population Center, University of North Carolina at Chapel Hill. Data collection for Add Health was supported by National Institute of Child Health and Human Development (NICHD) grant P01-HD31921; the revised documentation for public distribution was supported by NICHD grant 2 R44-HD31776. The opinions expressed in this manuscript are solely the authors'.

Author contact: dwiley@ucla.edu

\section{Coming Up in Perspectives}

To commemorate the 30th anniversary of Roe v. Wade, the January/February issue of Perspectives on Sexual and Reproductive Health will present the latest research on abortion in the United States, as well as several thought-provoking essays on what the legalization of abortion has meant and continues to mean to U.S. women and service providers. Here is what you will find in this special issue:

- "Abortion Incidence and Services in the United States in 2000," by Lawrence B. Finer and Stanley K. Henshaw

- "The Accessibility of Abortion Services in the United States, 2001," by Stanley K. Henshaw and Lawrence B. Finer

- "The Public Health Impact of Legal Abortion: 30 Years Later," by Willard Cates, Jr., David A. Grimes and Kenneth F. Schulz

- "Roe v. Wade at 30: What Are the Prospects for Abortion Provision?" by Carole Joffe

- "Abortion: Teaching Why as Well as How," by Felicia H. Stewart and Philip Darney

- "Beyond Apocalypse and Apology: A Moral Defense of Abortion," by Caitlin Borgmann and Catherine Weiss

- "Reflections of a Provider Before and After Roe: From the Voices of Choice Archive," Physicians for Reproductive Choice and Health

- "Convincing New Providers to Offer Medical Abortion: What Will It Take?" by Francine Coeytaux, Kirsten Moore and Lillian Gelberg 